\title{
Structural evolution of GaN during initial stage MOCVD growth
}

\section{Chong Cook Kim, Jung Ho Je, Min-Su Yi ${ }^{1}$, and Do Young Noh ${ }^{1}$}

Department of Materials Science and Engineering, Pohang University of Science and Technology, Pohang, Korea

${ }^{1}$ Department of Materials Science and Engineering and Center for Electronic Materials

Research, Kwangju Institute of Science and Technology, Kwangju, Korea

\begin{abstract}
The structural evolution of GaN films during the initial growth process of metalorganic chemical vapor deposition (MOCVD) - low temperature nucleation layer growth, annealing, and high temperature epitaxial growth - was investigated in a synchrotron $\mathrm{x}$-ray scattering experiment. The nucleation layer grown at $560^{\circ} \mathrm{C}$ that was predominantly cubic $\mathrm{GaN}$ consisted of tensile-strained aligned domains and relaxed misaligned domains. The hexagonal GaN, transformed from the cubic $\mathrm{GaN}$ during annealing to $1100^{\circ} \mathrm{C}$, showed disordered stacking. The atomic layer spacing decreased as the fraction of the hexagonal domains increased. Subsequent growth of epitaxial $\mathrm{GaN}$ at $1100{ }^{\circ} \mathrm{C}$ resulted in the formation of ordered hexagonal $\mathrm{GaN}$ domains with rather broad mosaicity.
\end{abstract}

\section{INTRODUCTION}

Gallium nitride $(\mathrm{GaN})$ is a direct wide-band-gap semiconductor that has attracted considerable interests in optoelectronic devices operating in blue-green to ultraviolet regime [1,2]. Device quality GaN films are currently grown by a two-stage metalorganic chemical vapor deposition (MOCVD) process wherein growth of $\mathrm{GaN}$ nucleation layers on sapphire (0001) substrates at a low temperature is followed by growth of epitaxial GaN films at a high temperature [2,3]. Resulting electrical, optical, and structural properties depend sensitively on the conditions under which the nucleation layer is processed $[2,4]$.

The structure of GaN films in the initial stage of the two-stage MOCVD growth is a focus of recent studies to elucidate the role of the nucleation layer in the final film quality. It has been revealed that the cubic $\mathrm{GaN}$ (c-GaN) with $\mathrm{ABCABC}$ stacking sequence and the hexagonal $\mathrm{GaN}(\mathrm{h}-\mathrm{GaN})$ with $\mathrm{ABAB}$ stacking sequence coexist in the nucleation layers $[5,6]$. Upon annealing to a high temperature prior to the second stage growth, GaN nucleation layer mostly transforms into the h-GaN. The purpose of this study is to reveal the detailed structural changes accompanying the cubic to hexagonal transformation during the initial stage growth process.

\section{EXPERIMENTAL DETAILS}

The GaN nucleation layers were grown on sapphire (0001) substrates using MOCVD. The substrates were cleaned with solvents and subjected to in-situ pretreatment under $\mathrm{H}_{2}$ flow at $1100^{\circ} \mathrm{C}$. The nucleation layers were grown at $560^{\circ} \mathrm{C}$ for 4 minutes using trimethylgallium (TMGa) and ammonia $\left(\mathrm{NH}_{3}\right)$. The annealed nucleation layers were obtained by heating the nucleation layers at $1100{ }^{\circ} \mathrm{C}$ for $6 \mathrm{~min}$ in the growth chamber. The epitaxial GaN was grown on the annealed nucleation layer at $1100^{\circ} \mathrm{C}$ for $5 \mathrm{~min}$. The $\mathrm{x}$ - 
ray measurements were performed $e x$-situ on the samples taken out of the growth chamber at the three different stages of the growth.

The synchrotron $\mathrm{x}$-ray scattering measurements were carried out at beamline $5 \mathrm{C} 2$ at Pohang Light Source (PLS) in Korea. The incident X-rays were focused vertically by a mirror. A double bounce $\mathrm{Si}(111)$ monochromator was used to monochromatize x-rays to the wavelength of $1.55 \AA$ and to focus the beam in horizontal direction. Two pairs of slits in front of the detector provided an appropriate instrumental resolution of about $0.001 \AA^{-1}$ in reciprocal space. The experiment was carried out by measuring the x-ray scattering profiles along the $<000 l>$ direction and along the $<10 \overline{1} l>$ directions in reciprocal space using hexagonal coordinates. The detailed explanation of the scattering geometry is explained in Ref.6.

\section{RESULTS AND DISCUSSIONS}

We first examined the as-grown $\mathrm{GaN}$ nucleation layer deposited at $560{ }^{\circ} \mathrm{C}$.

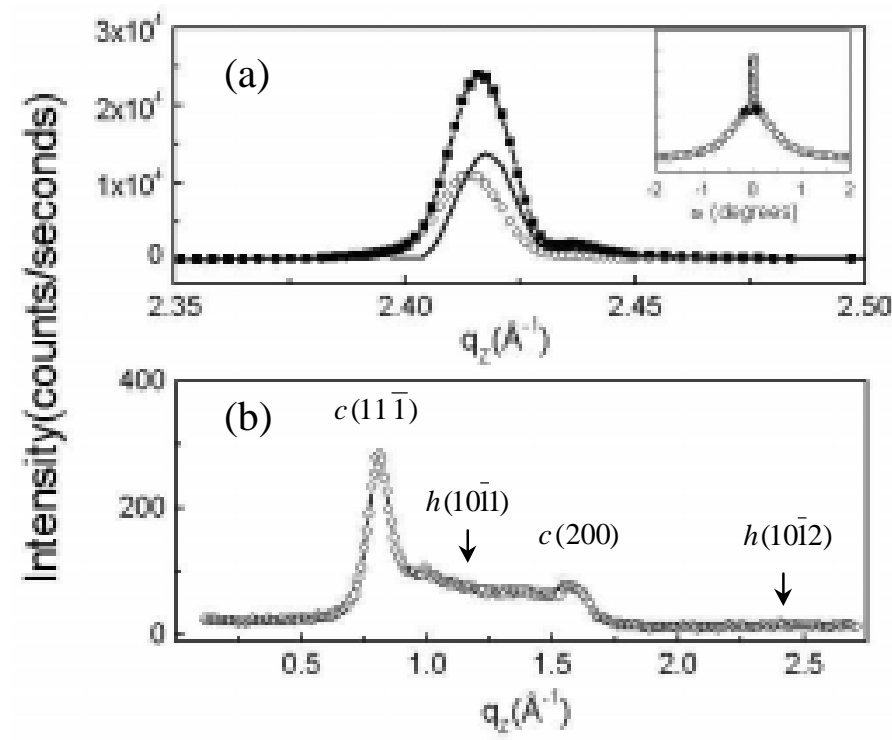

Figure 1. The $x$-ray diffraction profiles of the as-grown nucleation layer. (a) The longitudinal profiles of the $\mathrm{GaN}(0002)_{\mathrm{hex}}\left[\right.$ or the $\left.\mathrm{GaN}(111)_{\mathrm{cub}}\right]$ reflection. Solid squares represent the total intensity along the substrate normal direction, while the open circles represent the intensity measured at $0.1^{\circ}$ away from the normal direction. The solid line is the difference between the two that represents intensity from the aligned domains only. The inset of (a) represents the rocking curve of the peak. (b) The diffraction profile along the $\langle 10 \overline{1} l>$ direction. The peaks indicated by $c(11 \overline{1})$ and $c(002)$ are originated from $c$ -

GaN. 
Figures 1 (a) and its inset show the longitudinal profile and the rocking curve of the $\mathrm{GaN}(0002)_{\text {hex }}$ [or the $\mathrm{GaN}(111)_{\text {cub }}$ ] Bragg reflection, respectively. The rocking curve was composed of a sharp component and a broad component with $0.03^{\circ}$ and $1^{\circ}$ full width at half maximums (FWHM) respectively, indicating that there are domains with the crystalline axis aligned to the substrate normal direction (aligned domains) and domains with misaligned crystalline orientations (misaligned domains). The origin of the sharp component was attributed to the fact that the orientation of the domains grown in the very initial stage was constrained by the well-defined orientation of the sapphire substrate. Consequently the aligned domains are likely to be strained, while the misaligned domains are relaxed.

To examine the lattice strain of the as-grown nucleation layer, it is instructive to compare the c-axis lattice spacing of the aligned domains and that of the misaligned domains. The open circle in figure 1 (a) represents the diffraction profile of the misaligned domains measured at the rocking angle of $0.1^{\circ}$ away from the peak in the rocking curve. The solid line represents the profile from the well-aligned domains calculated by subtracting the diffraction signal of the misaligned-domains from the total signal indicated by the filled squares. The c-axis layer spacing of the aligned and the misaligned domains calculated from the peak positions using $l_{\mathrm{C}}=2 \pi / \mathrm{q}_{\mathrm{PEAK}}$ are $2.599 \AA$ and $2.603 \AA$ respectively. We note that the atomic layer spacing of the aligned domains, which are presumably more strained, is smaller than that of the misaligned domains by about $0.2 \%$. The in-plane lattice spacing of the aligned domains is presumably larger than that of the misaligned domains due to the effect of the usual tetragonal distortion [7]. This indicates that the well-aligned domains are under tensile stress in the film plane, which is counter-intuitive considering that the in-plane lattice spacing of the sapphire substrate is smaller than that of $\mathrm{GaN}$.

The tensile strain of the GaN nucleation layer can be understood in terms of the extended domain matching argument [8], wherein six Ga distances in $\mathrm{GaN}$ match to seven $\mathrm{Al}$ distances in sapphire. Although the in-plane lattice spacing of sapphire is smaller than that of $\mathrm{GaN}$, seven times the $\mathrm{Al}$ distance $(19.229 \AA)$ in sapphire is larger than six times the Ga distance $(19.134 \AA$ ) in $\mathrm{GaN}$ by $0.5 \%$. The film would then be under tensile stress. The tensile strain of about $0.2 \%$ observed in the aligned nucleation layer indicates that the strain was partially relaxed. The extended domain matching condition, however, would be satisfied only when the mobility of growing $\mathrm{GaN}$ is large enough to form reasonably large in-plane domains, which is controlled by the growth temperature of the nucleation layer.

Interestingly the c-axis lattice constants of both the aligned and the misaligned domains estimated from figure 1(a) are larger than the bulk hexagonal GaN lattice spacing, although the film was under tensile stress in the film plane. We attribute the increase of the c-axis lattice constant to the cubic stacking sequence between the atomic $\mathrm{GaN}$ layers. It has been suggested that the layer spacing of $\mathrm{c}-\mathrm{GaN}$ is larger than that of $\mathrm{h}$ $\mathrm{GaN}$ [9]. In fact, the diffraction profile along the $\langle 10 \overline{1} l>$ direction illustrated in figure 1(b) shows that the as-grown nucleation layer has the cubic stacking order. The reflections peaked at $\mathbf{q}_{\mathrm{Z}}=0.81 \AA^{-1}$ and $\mathbf{q}_{\mathrm{Z}}=1.56 \AA^{-1}$ correspond to the cubic $(11 \overline{1})$ and the cubic (002) Bragg reflections respectively. The correlation length of the stacking order estimated from the width of the peak is $78 \AA$.

As the nucleation layer was annealed to $1100{ }^{\circ} \mathrm{C}$, the stacking sequence changes from the cubic to hexagonal stacking as indicated by the appearance of the hexagonal 


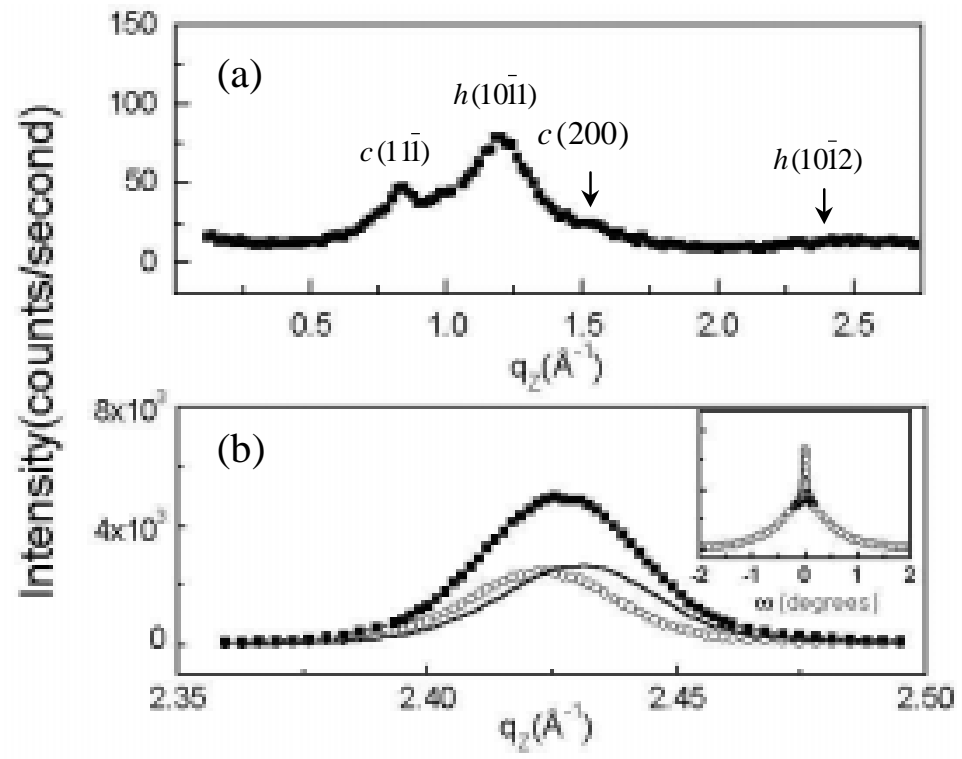

Figure 2. The $x$-ray diffraction profiles of the annealed GaN nucleation layer. (a) The diffraction profile along the $<10 \overline{1} l>$ direction. $\left.H_{(10} \overline{1} 1\right)$ represents the peak from $h-G a N$. (b) The longitudinal profiles of the GaN(0002). Solid squares represent the total intensity along the substrate normal direction, while the open circles represent the intensity measured at $0.1^{\circ}$ away from the normal direction. The solid line is the difference between the two that represents the intensity from the aligned domains only. The inset of $(b)$ represents the rocking curve at the peak.

(101) Bragg reflection at $q_{z}=1.2 \AA^{-1}$ in the diffraction profile shown in figure 2(a). The intensity of the cubic $(11 \overline{1})$ and the (002) reflections was greatly reduced, although they were still existent. The hexagonal (10 $\overline{1} 1)$ reflection was relatively broad, indicating that the correlation length of the stacking order was rather short, $\sim 24 \AA$. This suggests that the transformation was kinetically limited and many stacking faults were generated during the transformation.

The c-axis lattice constant decreased as the hexagonal stacking sequence developed during the annealing process as indicated by the shift of the GaN (0002) peak to a larger value in figure 2 (b). This is consistent with the fact that the layer spacing of the as grown c-GaN nucleation layer was larger than the bulk layer spacing of hexagonal GaN. The rocking curve of the peak shown in the inset of figure 2(b) demonstrates that the annealed $\mathrm{GaN}$ layer also consists of the aligned and the misaligned domains. By decomposing the longitudinal scan into two components similar to the case of the asgrown nucleation layer, we found that the aligned domains of the annealed $\mathrm{GaN}$ were still under tensile stress. The layer spacing of the aligned and misaligned domains was 2.586 $\AA$ and $2.593 \AA$ A respectively. 


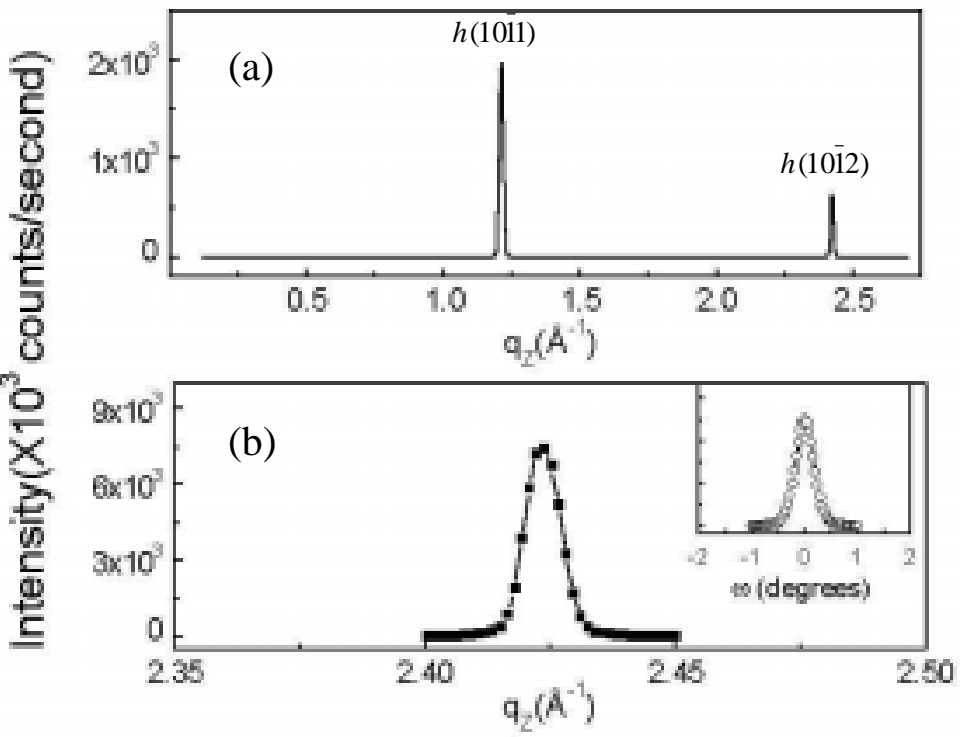

Figure 3. The x-ray diffraction profiles of the epitaxial GaN grown at $1100^{\circ} \mathrm{C}$ on the annealed GaN nucleation layer. (a) The diffraction profile along the $<10 \overline{1} l>$ direction. (b) The powder diffraction profiles in the $<000 l>$ direction. The inset of $(b)$ represents the rocking curve of the $\mathrm{GaN}(0002)$.

From the similarities between the rocking curves of the as-grown and the annealed sample, we conclude that the aligned c-GaN domains transformed to the aligned h-GaN and the misaligned c-GaN domains transformed to the misaligned h-GaN. We note that the longitudinal profile of the annealed sample [figure 2(b)] is broader than that of the asgrown sample [figure 1(b)]. This is another indication that the transformation was kinetically limited, and the remaining cubic stacking sequences acted as the faults in hexagonally stacked $\mathrm{GaN}$ layers.

The epitaxial GaN grown at $1100{ }^{\circ} \mathrm{C}$ for 5 min on the annealed $\mathrm{GaN}$ nucleation layers was completely hexagonal. The diffraction profile in the $\langle 10 \overline{1} l>$ direction illustrated in figure 3(a) shows only the hexagonal (10 $\overline{1} 1$ ) reflection without any trace of the cubic reflections. This suggests that the remaining cubic $\mathrm{GaN}$ in the annealed nucleation layer completely transformed to hexagonal $\mathrm{GaN}$ during the high temperature growth of the eptiaxial GaN. The peak was quite sharp and the correlation length of the stacking order was as large as $450 \AA$ indicating that the hexagonal stacking in the epitaxial $\mathrm{GaN}$ was well ordered.

In the meanwhile, the rocking curve of the $\mathrm{GaN}(0002)$ reflection illustrated in the inset of figure $3(\mathrm{~b})$ shows a single peak with $0.35^{\circ} \mathrm{FWHM}$. The mosaic distribution was broader than that of the aligned domains, but sharper than that of the misaligned domains of the nucleation layer. We believe that the epitaxial $\mathrm{GaN}$ nucleated on the misaligned domains whose mosaicity improved during the lateral growth at high temperatures $[10,11]$. 


\section{CONCLUSIONS}

In conclusion, we have revealed the details of the structural evolution of GaN films during the initial process of MOCVD. The as-grown nucleation layer that was predominantly c-GaN consisted of tensile-strained aligned domains and relaxed misaligned domains. The tensile strain of the $\mathrm{GaN}$ nucleation layer was explained by the extended domain matching argument. During annealing to $1100^{\circ} \mathrm{C}$, the $\mathrm{c}-\mathrm{GaN}$ transformed into disordered $\mathrm{h}-\mathrm{GaN}$. The atomic layer spacing decreased with increasing the fraction of the hexagonal domains, while the mosaic structure remained similar. The epitaxial $\mathrm{GaN}$ at grown $1100^{\circ} \mathrm{C}$ on the annealed nucleation layer was ordered hexagonal $\mathrm{GaN}$ domains with rather broad mosaicity.

\section{ACKNOWLEDGEMENTS}

This study was supported by Korean Research Foundation made in the program year of 1998, and by KOSEF through ASSRC (1998). PLS is supported by Korean Ministry of Science and Technology.

\section{REFRERENCES}

1. H. Morkoc and S. N. Mohammad, Science 267, 51 (1995)

2. S. Nakamura, Jpn. J. Appl. Phys. 30, L1705 (1991).

3. H. Amano, N. Sawaki, Akasaki, and Y. Toyoda, Appl. Phys. Lett. 48, 353 (1986)

4. S. Keller, B. P. Keller, Y. -F. Wu, B. Heying, D. Kapolnek, J. S. Speck, U. K. Mishra, and S. P. DenBaars, Appl. Phys. Lett. 68, 1525 (1996).

5. X. H. Wu, D. Kapolnek, E. J. Tarsa, B. Heying, S. Keller, B. P. Keller, U. K. Mishra, S. P. DenBaars, and J. S. Speck, Appl. Phys. Lett. 68, 1371 (1996).

6. A. Munkholm, C. Thompson, C. M. Foster, J. A. Eastman, O. Auciello, G. B. Stephenson, P. Fini, S. P. DenBaars, and J. S. Speck, Appl. Phys. Lett. 72, 2972 (1998)

7. D. Y. Noh, Y. Hwu, J. H. Je, M. Hong, and J. P. Mannaerts, Appl. Phys. Lett. 68, 1528 (1996)

8. N. Grandjean, J. Massies, P. Vennegues, M. Laugt, and M. Leroux, Appl. Phys. Lett. 70, 643 (1997)

9. T. Kurobe, Y. Sekiguchi, J. Suda, M. Yoshimoto, and H. Matsunami, Appl. Phys. Lett. 73, 2305 (1998)

10. X. H. Wu, P. Fini, S. Keller, E. J. Tarsa, B. Heying, U. K. Mishra, S. P. DenBaars, and J. S. Speck, Jpn. J. Appl. Phys. 35, L1648 (1996).

11. B. Yang, A. Trampert, B. Jenichen, O.Brandt, and K. H. Ploog, Appl. Phys. Lett. 73, 3869 (1998). 